\title{
KINETICS OF STRAIN RELAXATION IN CRYSTALLINE AND AMORPHOUS SOLID STATES
}

\author{
M. Duś-SiteK \\ Institute of Physics, Technical University of Częstochowa \\ Al. Armii Krajowej 19, 42-200 Częstochowa, Poland
}

\begin{abstract}
Strain recovery measurements under linear heating for Fe- and Co-based metallic glasses during axial tension of the hydrided crystalline $\mathrm{Ni}$ are presented. Using the mechanical, exoelectron emission and X-ray analysis methods the temperature of a start of high-rate strain recovery in metallic glasses was determined. The important role of the surface layer in the strains recovery processes of crystalline- $\mathrm{Ni}$ was shown.
\end{abstract}

PACS numbers: $79.75 .+\mathrm{g}$

\section{Introduction}

The process of continuous casting from the melt of metallic glasses is connected with structural relaxation during testing. Such quenching is usually interpreted according to the free-volume models developed by Spaepen [1] and Argon [2]. These models assume that a plastic deformation rate under given conditions is determined by the average free-volume. Annealing of the amorphous ribbons decreases the free volume which results in the lowering deformation rate. In the absence of an external load shear rearrangements are random and no macroscopic deformation occurs, but loading changes the activation barriers of these rearrangements processes so that structural relaxation becomes anisotropic (polarized by applied shear stress [3]) and leads to a macroscopic plastic deformation. One should consider the effect of an applied stress orientation on the elementary relaxation events.

The aim of this paper is to describe the kinetics of strain relaxation in amorphous and crystalline solid materials. This work attempts to analyze the experimental data in terms of surface layer models.

\section{Experiment and results}

Metallic glasses $\mathrm{Fe}_{78} \mathrm{Si}_{11} \mathrm{~B}_{11}$ and $\mathrm{Co}_{78} \mathrm{Si}_{11} \mathrm{~B}_{11}$ were heated to the required temperature, $T_{\mathrm{d}}$, inelastically deformed at this temperature to the proof stress $\varepsilon_{0}=$ $0.2 \%$ [4], cooled in the stressed state to room temperature, and finally unloaded. During this operations the induced internal stresses were measured. 


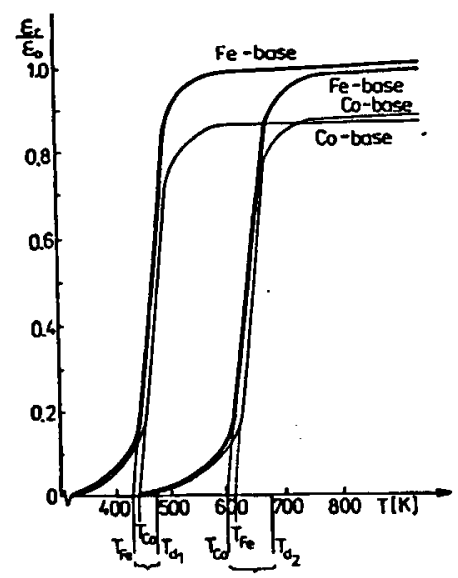

Fig. 1. Strain recovery curves for Fe- and Co-based metallic glasses predeformed ( $\varepsilon=$ $0.2 \%)$ at $T_{\mathrm{d} 1}=473 \mathrm{~K}$ and $T_{\mathrm{d} 2}=673 \mathrm{~K}$.

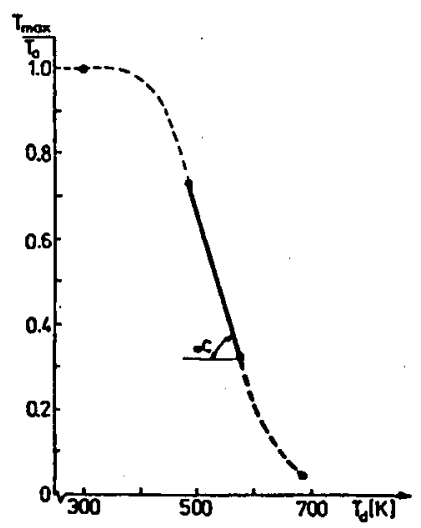

Fig. 2. The relationship of relative shift of relaxation maximum versus temperature $T_{d}$.

Results obtained for the temperatures $T_{\mathrm{d}}=473 \mathrm{~K}$ and $673 \mathrm{~K}$ are shown in Fig. 1. At $T<T_{\mathrm{d}}$ the recovery of deformation is small but its rate increases sharply and becomes maximal near $T_{\mathrm{d}}$. At $T \geq T_{\mathrm{d}}$ the recovery rate becomes close to zero (the degree of recovery, $\varepsilon_{\tau} / \varepsilon_{0} \approx 0.85 \div 1.0$ ). The measured intensity exoelectron emission as a function of temperature curves for these metallic glasses which have undergone the above described operations show that one of the maxima (maximum $T_{\text {relax }}$ ) [5] shifts sharply in the direction of low temperatures. At the same time the emission intensity at maximum decreases significantly. For the maximum assigned to structural relaxations the dependence of a relative size $T_{\max } / T_{0}\left(T_{0}-\right.$ temperature of this maximum for as-made specimen) on temperature $T_{\mathrm{d}}=473,573$ and $673 \mathrm{~K}$ (Fig. 2) was presented. There is probably a correlation between the rate of temperature lowering this maximum and the high-rate stress relaxation. 


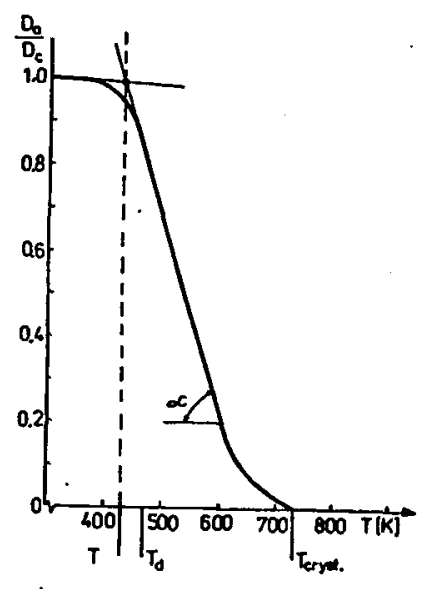

Fig. 3. The relationship of amorphous degree versus temperature for Co-based metallic glass predeformed $(\varepsilon=0.2 \%)$ at $T_{\mathrm{d}}=473 \mathrm{~K}$.

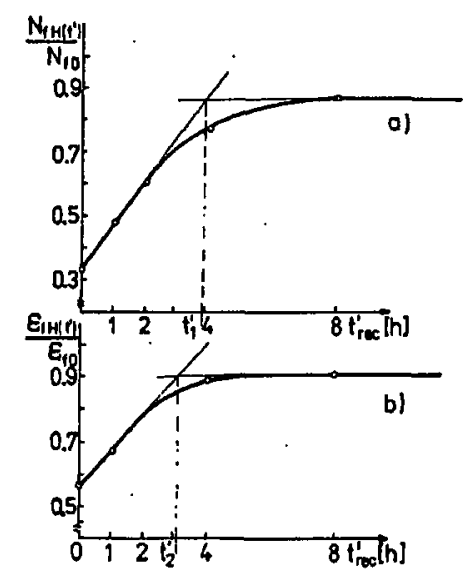

Fig. 4. The relationships of relative exoelectron intensities (a) and relative strains (b) versus recovery time, for crystalline hydrogenated $\mathrm{Ni}$.

Simultaneously, the degree of amorphization and crystallization of metastable phases for $\mathrm{Co}_{78} \mathrm{Si}_{11} \mathrm{~B}_{11}$ was determined using an X-ray diffraction technique. The relationship between the fraction of amorphous $D_{\mathrm{a}}$ and crystalline $D_{\mathrm{c}}$ phases versus temperature, calculated from experimental data [6] is presented in Fig. 3. Analogous results were obtained for another metallic glass investigated.

It can be noticed that the slopes of the curves in Fig. 2 and Fig. 3 are identical. Moreover, crystalline nickel was hydrided by cathodic saturation $\left(t_{\mathrm{s}}=\right.$ $24 \mathrm{~h}$ ) in pure $0.1 \mathrm{n}_{2} \mathrm{SO}_{4}$ and the exoelectron emission (EEE) measurements were performed after recovery times of $t_{\text {rec }}=0,1,2,4,8 \mathrm{~h}$ [7]. The EEE intensity curves ( $N$ versus deformation time $t$ ) show a typical characteristic, where $N_{f_{0}}$ and $\varepsilon_{f_{0}}$ are denoted for non-hydrided sample at the time of failure. The hydriding of the $\mathrm{Ni}$ 
sample caused changes in sample failure due to tension and also in EEE intensity $N_{f H}$ [7]. The value of sample-failure strain $\varepsilon_{f H}$ changes as well. The dependence of the ratio $N_{f H}\left(t^{\prime}\right) / N_{f_{0}}$ and of the relative strain $\varepsilon_{f H\left(t^{\prime}\right)} / \varepsilon_{f_{0}}$, on the recovery time $t_{\text {rec }}^{\prime}$ is shown in Fig. 4. Clearly, there is a correlation between the increase in the EEE intensity and the rate of the hydriding stress relaxation. Hydrogen stresses determined by X-ray method were about $(78.5-98) \times 10^{4} \mathrm{~N} / \mathrm{m}^{2}\left(10 \mathrm{kG} / \mathrm{m}^{2}\right)$.

\section{Discussion}

The character of the data in Fig. 1 suggests a qualitative model [8] which illustrates the sequential stages of homogeneous deformation and a subsequent recovery. Let any two states be small areas of structure in which under the joint action of temperature, $T_{\mathrm{d}}$, and applied stress, $\sigma$, elementary irreversible shears are possible. Cooling in the stressed state to room temperature results in "freezing" of their configuration. Subsequent unloading leads to formation of internal microstresses in area located between them, which are opposite to the external load. Upon heating this area under the combined action of internal stresses and thermal fluctuations can undergo shear with activation energy $E_{0}$. This shear will result in partial strain recovery. Similar direct processes will occur until their driving force, a distribution of internal stresses created by predeforming, is exhausted. The total orientation of these elementary shears will lead to complete strain recovery. Under continued heating, elementary shears will occur in a random, undirected stress field and the total deformation caused by them will be zero.

It can be considered that the sample was preannealed at $T=T_{\mathrm{d}}$ during $t=t_{\mathrm{d}}$; then at the time $t_{\mathrm{d}}$ the sample was loaded instantaneously to produce a plastic deformation of both regions and after that it was cooled down to room temperature. During subsequent heating of the cooled and unloaded sample, the structure will be "frozen" until reaching the temperature $T$, which corresponds to energy $E_{0}$.

It can be seen (Fig. 1) that high-rate strain recovery begins near this temperature $\left(T<T_{\mathrm{d}}\right)$. Experimental values of temperatures for the onset of recovery (which were determined as it is exemplified by the dashed line in Fig. 1) are also marked.

Thermostimulated exoelectron emission (TSEE) phenomenon and crystallization processes are connected with internal stresses and these relaxations [5]. The character of the data in Fig. 2 and Fig. 3 indicated that the stress relaxation is determined by irreversible shear processes with distributed activation energy [9] (stress relaxation for as-made specimens is defined by processes with activation energies lower than those for processes responsible for stress relaxation after preannealing at $T_{\mathrm{d}}$ ).

Considering changes of activation energy of unloaded samples, we can see that structural relaxations acquire a direct character and result in microscopic inelastic deformation. A decrease of activation barriers of favourably oriented relaxation centres will result at the beginning of high-rate stress relaxation. Therefore, the temperature $T$ of the onset of high-rate relaxation can be determined as shown by the dashed lines in Fig. 2 and Fig. 3. It can be assumed that these temperatures are determined mainly by annealing and should be independent of 
the chemical composition. So, stress relaxation can be interpreted as a result of a complete irreversible local atomic rearrangement. The experiment and discussion show that macroscopically reversible and irreversible deformation may be caused by irreversible anisotropic structural relaxation.

For the crystalline solid state (Ni) at $t^{\prime} \leq t_{2}^{\prime}$ (Fig. 4b) the recovery rate of strains, which was caused by hydrogen induced stresses, increases. However, at $t^{\prime}>t_{2}^{\prime}$ the recovery rate becomes close to zero (the degree of recovery rate $\approx 0.91 \div 1.0$ ). Because the strain after recovery time about $4-5 \mathrm{~h}$ reaches values of strain for non-hydrided samples we can say that strains, in this case, do not exceed the value of elastic limit (as suggested in Ref. [6]).

A comparison of Fig. 4a and Fig. 4b suggests that the failure deformation of a hydrogenated sample approaches the failure deformation $\left(\varepsilon_{f}\right)$ of a non-hydrogenated sample during shorter time than EEE values at the time of failure measured for the same sample. It can be explained by the fact that the sample failure starts from its surface, so the failure deformation is mainly influenced by the surface and the field which was induced over it ("supersurface"). Intensity EEE at the point of failure is connected with the electron emission from the superficial layer and from the inside of the sample. It seems that the observed phenomenon can be explained by hydrogen migration (the source of internal stresses) in the direction of the sample inside and at the sample surface.

At the initial stage of hydrogenation mainly compressive stresses are created in the superficial layer and their values do not exceed the yield point. So, the mechanism of hydrogen migration and changes of internal stress fields, explains the relation $t_{2}^{\prime}<t^{\prime}$.

For the times $t^{\prime \prime} \gg t_{1}^{\prime}$ and $t^{\prime} \gg t_{2}^{\prime}$ the same recovery rate of both mechanical (Fig. 4b) and emission (Fig. 4a) properties can be noticed (slopes of these lines are nearly the same). It can be assumed that even at the initial stage of hydrogenation, stresses in the superficial layer have not exceeded the yield point.

The fact that

(a) hydrogen (both in the gaseous and ionized state) is absorbed by $\mathrm{Ni}$ during electrolysis,

(b) the recovery rates of both mechanical and emission properties are similar for the times $t^{\prime} \gg t_{1}^{\prime}$ and $t^{\prime} \gg t_{2}^{\prime}$,

results in establishing the equilibrium conditions between hydrogen diffusion from the sample core to the superficial layer and hydrogen transport (changes of internal stresses) from the superficial layer to the outer region of the sample.

It seems that the layer facilitates the hydrogen penetration to the inside of the sample and makes difficult its transport to the outer part of the sample.

\section{References}

[1] A.J. Taub, F. Spaepen, Sci. Metall. 13, 195 (1979).

[2] A.S. Argon, Acta Metall. 27, 47 (1979).

[3] F. Spaepen, Physics of Defects, North-Holland, Amsterdam 1981, p. 136.

[4] A.G. Guy, Introduction to Materials Science, McGraw-Hill Book Company, New York 1971, p. 372. 
[5] M. Duś-Sitek, B. Wysłocki, Z. Olszowski, B. Sujak, Acta Phys. Pol. A 76, 123 (1989).

[6] M. Duś-Sitek, Acta Univ. Wratisl. 1264, 131 (1990).

[7] M. Duś-Sitek, Z. Olszowski, B. Sujak, J. Journ Appl. Phys. 24, 176 (1985).

[8] V.A. Kohnik, A.T. Kosilov, J. Non-Crystalline Solids 3, 270 (1994).

[9] M.R.J. Gibbs, J.E. Ivetts, J.A. Leak, J. Mater. Sci. 20, 2517 (1985). 